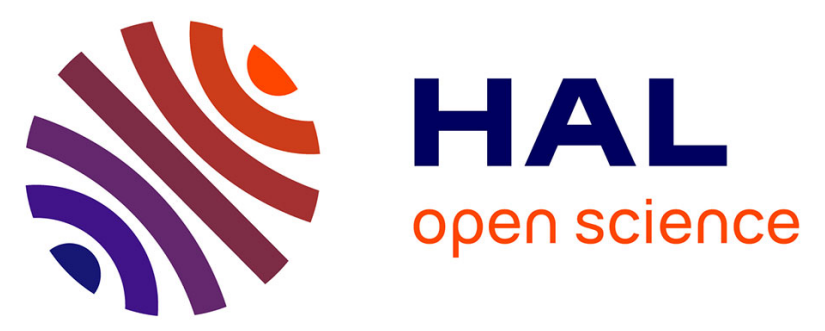

\title{
Impairment of Shooting Performance by Background Complexity and Motion
}

\author{
Loïc Caroux, Ludovic Le Bigot, Nicolas Vibert
}

\section{To cite this version:}

Loïc Caroux, Ludovic Le Bigot, Nicolas Vibert. Impairment of Shooting Performance by Background Complexity and Motion. Experimental Psychology, 2015, 62 (2), pp.98-109. 10.1027/16183169/a000277 . hal-01097375

\section{HAL Id: hal-01097375 \\ https://hal.inria.fr/hal-01097375}

Submitted on 19 Dec 2014

HAL is a multi-disciplinary open access archive for the deposit and dissemination of scientific research documents, whether they are published or not. The documents may come from teaching and research institutions in France or abroad, or from public or private research centers.
L'archive ouverte pluridisciplinaire HAL, est destinée au dépôt et à la diffusion de documents scientifiques de niveau recherche, publiés ou non, émanant des établissements d'enseignement et de recherche français ou étrangers, des laboratoires publics ou privés. 


\section{PRE PRINT}

Caroux, L., Le Bigot, L., \& Vibert, N. (in press). Impairment of shooting performance by background complexity and motion. Experimental Psychology. doi:10.1027/1618-3169/a000277

This article may not exactly replicate the final version published in Experimental Psychology (C) 2014 by Hogrefe Publishing. It is not the version of record and is therefore not suitable for citation.

Title:

Impairment of shooting performance by background complexity and motion

Authors:

Loïc CAROUX, Ludovic LE BIGOT, Nicolas VIBERT

Affiliation:

Centre de Recherches sur la Cognition et l'Apprentissage (CeRCA)

UMR 7295 - University of Poitiers / University of Tours / CNRS

France

(Loïc Caroux is currently affiliated to INRIA Bordeaux Sud-Ouest, Talence, France)

\section{Corresponding author:}

Loïc Caroux

INRIA Bordeaux Sud-Ouest

Equipe Phoenix

200 avenue de la Vieille Tour

33405 Talence Cedex

France

Email: loic.caroux@inria.fr 


\section{Abstract}

In many visual displays such as virtual environments, human tasks involve objects superimposed on both complex and moving backgrounds. However, most studies investigated the influence of background complexity or background motion in isolation. Two experiments were designed to investigate the joint influences of background complexity and lateral motion on a simple shooting task typical of video games. Participants had to perform the task on the moving and static versions of backgrounds of three levels of complexity, while their eye movements were recorded. The backgrounds displayed either an abstract (Experiment 1) or a naturalistic (Experiment 2) virtual environment. The results showed that performance was impaired by background motion in both experiments. The effects of motion and complexity were additive for the abstract background and multiplicative for the naturalistic background. Eye movement recordings showed that performance impairments reflected at least in part the impact of the background visual features on gaze control.

\section{Keywords}

Eye movements; Low-level visual features; Virtual environments; Visual perception 


\section{Introduction}

In virtual environments, most tasks involve the use of objects that are superimposed on a background. In general, these backgrounds are complex and dynamic as in natural scenes. They are composed of various visual information (e.g., building insides, landscapes) and can move in different ways (e.g., linear or circular motion) in connection with the task in which people are engaged (e.g., Riecke, Schulte-Pelkum, Avraamides, Von Der Heyde, \& Bülthoff, 2006; Trutoiu, Mohler, SchultePelkum, \& Bülthoff, 2009).

As detailed below, many studies have shown that background complexity (e.g., Chen \& Hegdé, 2012; Jie \& Clark, 2008; Wolfe, Oliva, Horowitz, Butcher, \& Bompas, 2002) or motion (e.g., Caroux, Le Bigot, \& Vibert, 2013; Harrison, Thompson, \& Sanderson, 2010; Honda, 2001; Kaminiarz, Krekelberg, \& Bremmer, 2007; Tozzi, Morrone, \& Burr, 2007) could impair people's performance in various tasks and situations. In general, the impairment can be explained by the people's gaze behavior, which reflects attentional processes (e.g., Henderson, Chanceaux, \& Smith, 2009; Ilg, 1997). However, few studies investigated the joint effects of background motion and complexity. The objective of the present experiments was to fill this gap by investigating the joint influences of background complexity and background motion on performance and gaze behavior in a virtual environment.

\subsection{Influence of background complexity on performance and gaze behavior}

The complexity of a visual scene is usually characterized by low-level visual features. To qualify the complexity of a visual scene, the concept of "clutter" can be used (e.g., Asher, Tolhurst, Troscianko, \& Gilchrist, 2013; Beck, Lohrenz, \& Trafton, 2010; Neider \& Zelinsky, 2011; Wolfe et al., 2002). The clutter represents the density of visual information in a scene. When the clutter of a visual scene is high, the scene includes a great density of various color or luminance contrasts. When the clutter is 
low, only few contrasts are visible in the scene. The complexity of a visual scene has an impact on people's performance and associated gaze behavior (Henderson et al., 2009) in various tasks, such as visual search (e.g., Wolfe et al., 2002) or video game shooting tasks (Jie \& Clark, 2008).

Wolfe et al. (2002) studied visual search in complex visual environments and proposed a model based on the "guided search" model (Wolfe, 1994). Searching for an item in a complex visual scene can be separated in two stages: the pre-attentive stage and the selective stage. In the first stage, parallel processing of visual information is used to guide the deployment of attention, and candidate targets are segmented from the background. In the second stage, attention is oriented towards the pre-selected items, which are processed further to identify the target. Wolfe et al. (2002) showed that the more complex the scene was, the more imperfect the pre-attentive segmentation was. In addition, the duration of processing of pre-selected items depended on the quality of the separation of items from the background. Hence, the more complex the visual environment was, the lower the observer's performance was and the slower the observers were to detect the target. Similarly, Jie and Clark (2008) studied the effect of background complexity on the perception of superimposed items in a shooting task. Participants had to detect targets that appeared randomly on a stationary complex picture, and to shoot them as rapidly as possible. The authors showed that performance (time to shoot targets) was lower when the local clutter around the target was higher.

The time to process a display can also be assessed by analyzing observers' eye movements (for a review, see Rayner, 2009). For example, the duration of searchers' eye fixations is often used to quantify the amount of attentional resources required to process visual items in the environment. Henderson et al. (2009) showed that in a real world scene, the more complex the scene was, the higher the mean duration of eye fixations was. This was coherent with Wolfe et al.'s (2002) findings about the time needed to detect the target during a visual search task performed on a complex background. This reflected the difficulty for the observer to extract useful information from the local 
visual "noise". Hence, the mean duration of eye fixations is an indicator of the search efficiency within a complex scene.

\subsection{Influence of background motion on gaze behavior and performance}

One of the best known effects of moving backgrounds in natural scenes or virtual environments is the optokinetic nystagmus (OKN) triggered by large-scale motions of the visual surroundings (e.g., Kim \& Palmisano, 2010; Riecke et al., 2006). The OKN is a reflexive, conjugate movement of both eyes in which two phases alternate: the slow phase moves the eyes in the direction of background motion, ideally at the same velocity, whereas the fast phase regularly brings back the eyes in the opposite direction (IIg, 1997; Waespe \& Schwarz, 1987). The slow phase of the OKN is a compensatory eye movement that allows the observer to automatically keep visual input stable on the retina. Even though the slow and fast phases tend to compensate each other, the average gaze orientation is generally diverted towards where the moving scene comes from, at least when the observer is passively looking at the scene (IIg, 1997). Note that any single, stationary fixation point presented on top of the moving background can be used by observers to voluntarily cancel the OKN (Ilg, 1997).

The OKN has behavioral implications for activities performed on moving visual backgrounds. For example, the OKN has negative effects on observers' performance in perceptive tasks. Kaminiarz et al. (2007) and Tozzi et al. (2007) studied the impact of OKN on participants' performance in a target localization task. Participants had to localize a briefly flashed target displayed on a laterally moving, patterned background. The authors showed that various errors of localization were observed during OKN phases. Caroux et al. (2013) studied the effect of background motion on the perception of superimposed items during a shooting task. Participants had to detect targets that appeared randomly on patterned backgrounds, and to shoot them as rapidly as possible. They showed that 
performance (time to shoot targets) was lower when the background was laterally moving than when it was stationary. Similarly, Harrison et al. (2010) demonstrated that background motion decreased performance in a simple task involving the integration of brief auditory and visual signals. The visual image was displayed on a head-mounted display and the background was the real world. When the participants walked or when the walls surrounding the participants moved, the performance was lower than when the participants sat or when the walls were stationary. In sum, moving artificial or real world backgrounds do often decrease performance in simple tasks such as target localization or target shooting tasks.

\subsection{The present study}

The present study aimed at investigating the joint influences of background lateral motion and visual complexity on a simple shooting task such as what can be found in video games. The goal of the shooting task used in the experiments was to aim and shoot as fast as possible at different visual targets indicated by auditory clues to obtain the best score possible. The first hypothesis was that the performance is lower when the background is visually complex than when it is less complex (Jie \& Clark, 2008; Wolfe et al., 2002). Because previous studies showed that background motion also impaired performance in target detection tasks (Caroux et al., 2013; Harrison et al., 2010; Kaminiarz et al., 2007; Tozzi et al., 2007), the performance impairment induced by visual complexity should interact with the impairment caused by background motion. Thus, the second hypothesis was that the performance is more impaired by background complexity when the background is moving than when it is static. These hypotheses were tested in two similar experiments. The only difference between the experiments was the nature of the visual elements used to design the background. The findings were expected to be the same whatever the background visual display. 


\section{Experiment 1}

\subsection{Method}

\subsubsection{Participants}

Twenty-two volunteers (16 women, 6 men) took part in the experiment. Their average age was 19.9 years $(S D=1.8)$ and their average length of schooling 13.6 years $(S D=1.4)$. All participants were native French speakers and had normal or corrected-to-normal vision.

\subsubsection{Apparatus}

A non-invasive, head-free "Tobii T120" eye-tracker that looked like a 17" TFT computer screen (1280 x 1024 pixels resolution) was used to mimic as much as possible natural interaction with a virtual environment. The eye tracker was controlled via a computer, which collected the data and ran the task program. Gaze positions were obtained at a $120 \mathrm{~Hz}$ frequency with an average precision of 0.5 degree of visual angle (about $5 \mathrm{~mm}$ on the screen). Because the laterally moving backgrounds used in the experiments moved leftwards at about $12 \mathrm{deg} / \mathrm{s}$ (see below), the data may include OKN slow phases during which the acquisition of information is still ongoing even though the observer's gaze is moving rightwards at the same velocity. Hence, eye fixations and saccades were detected using velocity-based detection algorithms from the "GazeAlyze" software (Berger, Winkels, Lischke, \& Höppner, 2012). Fixations were defined as any period where gaze velocity was less than $14 \mathrm{deg} / \mathrm{s}$ for at least $60 \mathrm{~ms}$. Saccades were defined as any period where gaze velocity went over $30 \mathrm{deg} / \mathrm{s}$ for more than 35 ms. A speaker that was located in front of participants (without bias of spatial location) was used to display sounds. 


\subsubsection{Material}

The material was created with "Adobe Director 11" software. Three different backgrounds of various visual complexities were designed (Figure 1). They were extracted from the video game "Child of Eden" (Ubisoft, 2011) and were composed of a mainly green neutral pattern (main HSV color scale: Hue $=120$, Saturation $=100$, Value $=10$ to 70$)$ scattered with abstract objects (HSV: $120,50-100,40-$ 100). The complexity was determined according to the proportion of the background covered by the objects, which represented the clutter. The first version only showed the neutral pattern (no clutter), the second one showed the neutral pattern with objects covering $25 \%$ of its surface (low clutter), and in the third one the objects covered $50 \%$ of the background surface (high clutter). As stated above, when the background was laterally moving, it moved leftwards at a speed of $121 \mathrm{~mm} / \mathrm{s}$ (about 12 $\mathrm{deg} / \mathrm{s})$. The target and the distractor of the shooting task were drawings of a creature. Four creature versions were used. The creature could be blue (HSV: 228, 74, 25-84) or red (HSV: 7, 74, 25-84), and small (85x66 pixels, 22x17 mm, 2,2×1,7 degrees) or large (85x99 pixels, 22×26 mm, 2.2x2.6 degrees). The cursor was a representation of a crosshair of 114 pixels diameter ( $30 \mathrm{~mm}, 3$ degrees). Six auditory clues were recorded with a male natural voice. These clues were "left", "right", "blue", "red", "large" and "small" ("gauche", "droite", "bleu", "rouge", "grand" and "petit" in French). Their duration was 0.5 second.

[Figure 1 near here]

\subsubsection{Design and procedure}

The background complexity (no, low or high clutter) and motion (presence or absence) were manipulated within-participants. 
A shooting task was used. At the beginning of each trial, the background was displayed and an empty black square of $74 \mathrm{~mm}$ side (about $7.4 \mathrm{deg}$ ) was transiently superimposed at the centre of the screen for 1 second to orient the participant's gaze towards the centre of the display without cancelling the effects of background motion such as the OKN. Participants were instructed to keep their gaze as much as possible within the square. The auditory clue was displayed at the same time than the square. The clue could be a location clue ("left" or "right"), a color clue ("blue" or "red") or a size clue ("large" or "small"). The target, the distractor and the cursor appeared on the screen all on the same, random horizontal line. The cursor was always on the central vertical axis of the screen, whereas the target was displayed randomly on the right or left side of the screen. The horizontal coordinate of appearance of the target (between the cursor and the screen side) was also randomly determined. The distractor was located at the exact opposite of the target on the other side of the screen central vertical axis. The size and color of the distractor differed from the target only on the visual feature that corresponded to the nature of the clue. For example, if the clue was "red", the target was necessarily red but could be large or small. The distractor was of the opposite color (here blue) but had the same size as the target. Participants had to aim at the target with the help of the keyboard buttons "left arrow" and "right arrow", and to shoot at it by pushing the "space" bar. Participants were able to move the cursor and shoot as often as they wished while the target was missed, in other words while the cursor and the target were not superimposed at the time of shooting. When the target was hit, another trial began.

The experimenter presented this procedure to the participants as a video game. They were asked to touch as fast as possible each target by neglecting the distractor. Each set of trials was presented to the participant as one game. Six games of sixty trials were presented to each participant, with a pause between each game. The global score was given at the end of all blocks. All trials of each game were performed on the same background, which did not disappear between each trial. When the background was moving, the motion was continuous and did not stop for the whole game. The order of presentation of the 6 games ( $2 \times 3$ different conditions of motion presence/absence and 
complexity) per participant was pseudo-randomized. In each game, the order of presentation of the different clues was also pseudo-randomized.

\subsubsection{Dependent measures}

Performance was assessed using the mean response time to hit each target (or to complete each trial) as the dependent measure. To explain the performance, participants' eye movements were analyzed using six dependent measures: the mean number of fixations per trial, the mean duration of fixations, the average location of fixations on the display (defined by the horizontal $-\mathrm{X}-$ and vertical $-Y-$ coordinates), the mean number of saccades per trial and the average amplitude of saccades. For each trial, only the eye fixations and saccades that were made while searching for the target. In other words, both the fixation that was interrupted by the appearance of the target and distractor and the fixation that was ongoing when the target was hit were not included in the analysis. All variables were analyzed using repeated measures ANOVAs with the background motion and the background complexity as within-participants factors. When the Mauchley sphericity test was significant, Greenhouse-Geisser correction was applied.

\subsection{Results}

All late responses (response times greater than $3000 \mathrm{~ms}$ ) were excluded from analyses. The number of trials that were excluded represented on average $2.1 \%$ of the total number of trials per participant $(\min =0.0 \%, \max =13.3 \%)$. Response time data were logarithmically transformed before ANOVAs were performed. For clarity of presentation, the means of the untransformed data are reported in the text, graphic and table. The means and standard deviations of all dependent measures are displayed in Table 1. The results of the ANOVAs and planned comparisons that were performed to analyze the data are displayed in Table 2 . 
[Table 1 near here]

[Table 2 near here]

\subsubsection{Mean response time}

As shown on Figure 2, background complexity had an influence on the response time. Planned comparisons demonstrated that the response time was longer in the low clutter condition $(M=1392$ $\mathrm{ms}, S D=264)$ than in the no clutter $(M=1341 \mathrm{~ms}, S D=253)$, or high clutter conditions $(M=1359 \mathrm{~ms}$, $S D=265)$. The difference between these two last conditions was not significant. Background motion had also an impact on the response time. The response time was longer on the moving background $(M=1404 \mathrm{~ms}, S D=244)$ than on the static one $(M=1324 \mathrm{~ms}, S D=272)$. However, the interaction between the motion and complexity factors was not significant.

[Figure 2 near here]

\subsubsection{Eye movement data}

Qualitative observation of the eye movement data displayed by a sample of participants showed that when the background was moving, an OKN consisting of slow phases oriented leftwards interrupted by quick phases oriented rightwards was present in between trials. The maximum velocity of slow phases reached 5 to $10 \mathrm{deg} / \mathrm{s}$ depending on participants. When the target and distractor appeared, the OKN disappeared, and only fixations and saccades were visible, probably because the target and/or distractor were used as stationary "anchoring points" to cancel the nystagmus (IIg, 1997). 
As explained in the method section, only the eye fixations and saccades that were made while searching for the target were analyzed. Hence, the fixations and saccades that were analyzed accounted for about $50 \%$ of response times.

\section{Fixations}

In accordance with the response time data, background complexity had an influence on the number of fixations that were made while searching for the target. Planned comparisons demonstrated that the number of fixations was higher in the low clutter condition than in the no clutter one. Other comparisons were not significant, even though participants tended to make more fixations in the low clutter condition than in the high clutter one. Background motion had also an impact on the number of fixations, which was higher on the moving background than on the static one. However, the interaction between the two factors was not significant.

Background motion had an impact on the horizontal coordinate $(X)$ of fixations. In average, fixations were located more rightwards on the moving background than on the static one. Background complexity had no impact on the horizontal coordinate of fixations, but the interaction between the two factors was significant. Planned comparisons demonstrated that when the background was moving, the fixations were located more rightwards in the high clutter condition than in the low clutter one, and also more rightwards in the low clutter condition than in the no clutter one. When the background was static, in contrast, there was not any significant effect of background complexity on the horizontal coordinate of fixations.

There was not any significant effect of background complexity or motion on the mean duration or the vertical coordinate $(\mathrm{Y})$ of the fixations, or any significant interaction between the two factors.

\section{Saccades}

The number of saccades made by the participants while searching for the target was higher, and their average amplitude lower, on the moving background than on the static one. There was not any 
significant effect of background complexity on the mean number or the average amplitude of saccades, or any significant interaction between the two factors.

\section{Experiment 2}

\subsection{Method}

The apparatus, design, procedure and participants of Experiment 2 were the same as those of Experiment 1. The material was the same as in Experiment 1, but the three backgrounds were extracted from the video game "Sim City 4" (Electronic Arts, 2003) (Figure 1), and were composed of a mainly green neutral pattern scattered (HSV: 75, 30-50, 30) with naturalistic objects (HSV: 75, 0-50, 20).

\subsection{Results}

As in experiment 1, all late responses (response times greater than $3000 \mathrm{~ms}$ ) were excluded from analyses. The number of trials that were excluded represented on average $2.0 \%$ of the total number of trials per participant $(\min =0.0 \%, \max =10.8 \%)$. Response time data were logarithmically transformed before ANOVAs were performed. For clarity of presentation, the means of the untransformed data are reported in the text, graphic and table. The means and standard deviations of all dependent measures are displayed in Table 3. The results of the ANOVAs and planned comparisons that were performed to analyze the data are displayed in Table 4.

[Table 3 near here]

[Table 4 near here] 


\subsubsection{Mean response time}

As shown on Figure 3, background complexity had not any significant influence on the response time. In contrast, background motion had an impact on the response time, which was longer on the moving background $(M=1360 \mathrm{~ms}, S D=262)$ than on the static one $(M=1291 \mathrm{~ms}, S D=250)$. Furthermore, the interaction between background motion and complexity was significant. Planned comparisons showed that when the background was moving, the response time was longer in the low clutter condition than in the no clutter one. Other comparisons were not significant. When the background was static, the response time was not significantly different between the three clutter conditions.

[Figure 3 near here]

\subsubsection{Eye movement data}

As in Experiment 1, qualitative observation of the eye movement data displayed by a sample of participants suggested that when the background was moving, an OKN was triggered in between trials. Again, only the eye fixations and saccades that were made while searching for the target were analyzed. They accounted for about $40 \%$ of response times.

\section{Fixations}

Even though participants tended to make more fixations when the background was moving, there was not any significant effect of background complexity or motion on the mean number of fixations that were made while searching for the target, or any significant interaction between the two factors. 
Similarly, there was not any significant effect of background complexity or motion on the mean duration or the vertical coordinate $(\mathrm{Y})$ of the fixations, or any significant interaction between the two factors

As in Experiment 1, background motion had an impact on the horizontal coordinate $(X)$ of fixations. The fixations were located more rightwards on the moving background than on the static one. In contrast, there was not any significant effect of background complexity on the horizontal coordinate of fixations, or any significant interaction between the two factors.

\section{Saccades}

As in Experiment 1, the average amplitude of saccades was lower on the moving background than on the static one. But, in contrast, there was not any significant effect of background motion on the mean number of saccades. There was not any significant effect of background complexity on the mean number or the amplitude of saccades, or any significant interaction between the two factors.

\section{Discussion}

The first hypothesis was that the performance is lower when the background is complex than when it is less complex. It was partially supported. The response time varied significantly according to the background complexity only in Experiment 1. Furthermore, the response time was higher in the low clutter condition than in both the no clutter and high clutter conditions. In accordance with these data, participants made more or tended to make more fixations while searching for the target in the low clutter condition than in the no clutter or high clutter conditions. According to Beck et al. (2010) and Wolfe et al. (2002), the performance impairment in a visual search task would linearly increase with the visual complexity of the scene. Thus, the participants' response time should have been higher in the high clutter than in the low clutter condition. Hence the way the visual complexity of backgrounds was set in the present study may not have been completely adequate. According to the results, the presence of objects superimposed on a pattern does increase the visual complexity of the 
background, but increasing the proportion of the surface they cover does not seem to increase background complexity, and may actually decrease it. The quantity of salient visual contrasts in the background may not directly depend on the proportion of the scene covered by the objects.

In order to have another measure than the level of clutter to qualify the complexity of each background used in the present study, saliency maps were computed from the various backgrounds used in the two experiments using the "Saliency toolbox 2.3" (Walther \& Koch, 2006), which implements the saliency model originally proposed by Itti and Koch (2000) (Figure 4). This model is able to predict human gaze allocation on the different parts of visual scenes by computing regions of saliency. In the resultant saliency maps, low values indicate the regions of the scene that are not salient, whereas high values indicate the regions that are salient and tend to attract the observer's attention.

Qualitative observation of the saliency maps obtained for the backgrounds used in Experiment 1 show that there were several highly salient regions in the low clutter background, whereas the overall saliency of salient regions was lower in the no clutter and high clutter backgrounds.

Consequently, the participants' gaze was probably more impacted by low-level visual features in the low clutter background than in the other ones. This is in accordance with response time data, which demonstrated that performance was more impaired by the low clutter background than by the high clutter one.

The saliency maps obtained for the backgrounds used in Experiment 2 were quite similar to those obtained in Experiment 1, i.e. displayed more highly salient regions in the low clutter condition than in the two other ones. However, the difference between the three types of background was less important than in Experiment 1. This could explain why the participants' gaze and performance were not significantly impacted by manipulations of background complexity, but only by the joint manipulation of both background motion and complexity. 
[Figure 4 near here]

In sum, in these two experiments, the most visually complex background was apparently that where the superimposed objects covered $25 \%$, and not $50 \%$, of the screen surface. When the proportion of the screen covered by objects was too high, the impact of the background was not significantly different from that of the basic background where no object was present. Hence, the saliency scale of a visual scene seems to give a better measure of complexity than the level of clutter, but complementary experiments are needed to determine to what extent this is true.

Contrary to what was expected according to Henderson et al. (2009), the analysis of eye movements did not demonstrate any significant influence of background complexity on the duration of eye fixations. This confirms that the proportion of the scene covered by objects may not be an ideal measure of background complexity. Alternatively, the influence of this factor on the duration of fixations may be too weak in the present conditions to be observable.

The second hypothesis was that with a complex background, the performance is even more impaired when, in addition, the background is moving. It was partially supported. The results showed that the performance was always lower when the background was moving than when it was static, whatever the type of background. These results are in line with those of previous studies (Caroux et al., 2013; Harrison et al., 2010; Kaminiarz et al., 2007; Tozzi et al., 2007). The analysis of eye movements showed that this impairment may be due to the need to cancel the OKN triggered by background motion, which would disturb gaze control and information processing while the participants are searching for and shooting at the target. Indeed, when the background was moving, the average gaze orientation was deviated towards the side of origin of the motion as expected in the presence of OKN (Ilg, 1997). In addition, participants made smaller saccades in both experiments and tended to make more saccades and fixations on the display, though significance was reached only in Experiment 1. 
Contrary to predictions, however, the predicted interaction between the background motion and complexity was significant only in Experiment 2. The results showed that when there was no clutter or a high clutter, there was not any significant difference of response time between the static and moving backgrounds. When the clutter was low, the response time was higher on the moving background than on the static one. In this experiment, the presence of objects superimposed on the neutral pattern impaired performance only when the background was moving, and only when the proportion of the background surface covered by objects was not too high (i.e. when the background included numerous highly salient regions).

\subsection{Conclusions and outlook}

The present study showed that the performance in a simple shooting task is often impaired by background motion, in accordance with previous studies with other tasks (Caroux et al., 2013; Harrison et al., 2010; Kaminiarz et al., 2007; Tozzi et al., 2007). Moreover, the present study demonstrated that the effects of background visual complexity and motion on the performance are additive for one of the backgrounds (Experiment 1 ) and multiplicative for the other one (Experiment 2).

However, one limit of the present study is that the two experiments did not give exactly the same results. The impact of background complexity on participants' performance was different according to the background that was used. In Experiment 1, the simple effect of background complexity was significant. In Experiment 2, the simple effect of background complexity was not significant, but there was a significant interaction with background motion such that background complexity had a significant impact on performance when the background was moving.

This difference of results can only be due to the nature of the visual elements that were used in the two backgrounds. All other parameters of the experiments were strictly similar. Among possible explanations, the different arrangements of the objects on the neutral pattern, or the different 
shades of greens used for the patterns, the objects or both seem to create differential contrasts and local complexities between the two backgrounds. In addition, the colors of the different visual elements used in the two backgrounds were different in terms of color "hue", "saturation" and "value". All this may influence the difficulty of the target and distractor perception task. For example, previous studies showed that visual search performance may vary depending on other types of lowlevel features of the background, such as luminance, orientation and spatial frequency (e.g., De Vries, Hooge, Wertheim, \& Verstraten, 2013). Further experiments are necessary to explain to what extent these specific low-level features can influence the perception and use of objects superimposed on a structured background, in combination with background complexity and motion.

\section{Acknowledgments}

The authors thank Jean Pylouster for his technical assistance in the analysis of the eye movement data, especially in the adaptation of the "GazeAlyze" software package for the Tobii T120 data. Loïc Caroux was supported by a Ph.D. fellowship from the Direction Générale de l'Armement (DGA, French Ministry of Defense) and followed in the frame of this fellowship by Didier Bazalgette.

\section{References}

Asher, M. F., Tolhurst, D. J., Troscianko, T., \& Gilchrist, I. D. (2013). Regional effects of clutter on human target detection performance. Journal of Vision, 13(5), article 25, 1-15. doi:10.1167/13.5.25

Beck, M. R., Lohrenz, M. C., \& Trafton, J. G. (2010). Measuring search efficiency in complex visual search tasks: Global and local clutter. Journal of Experimental Psychology: Applied, 16, 238250. doi:10.1037/a0019633

Berger, C., Winkels, M., Lischke, A., \& Höppner, J. (2012). GazeAlyze: a MATLAB toolbox for the 
analysis of eye movement data. Behavior Research Methods, 44, 404-419.

doi:10.3758/s13428-011-0149-x

Caroux, L., Le Bigot, L., \& Vibert, N. (2013). Impact of the motion and visual complexity of the background on players' performance in video game-like displays. Ergonomics, 56, 1863-1876. doi:10.1080/00140139.2013.847214

Chen, X., \& Hegdé, J. (2012). Learning to break camouflage by learning the background. Psychological Science, 23, 1395-1403. doi:10.1177/0956797612445315

De Vries, J. P., Hooge, I. T. C., Wertheim, A. H., \& Verstraten, F. A. J. (2013). Background, an important factor in visual search. Vision Research, 86, 128-138. doi:10.1016/j.visres.2013.04.010

Harrison, W. J., Thompson, M. B., \& Sanderson, P. M. (2010). Multisensory integration with a headmounted display: Background visual motion and sound motion. Human Factors, 52, 78-91. doi:10.1177/0018720810367790

Henderson, J. M., Chanceaux, M., \& Smith, T. J. (2009). The influence of clutter on real-world scene search: Evidence from search efficiency and eye movements. Journal of Vision, 9(1), article 32, 1-8. doi:10.1167/9.1.32

Honda, H. (2001). Visual mislocalisation induced by translational and radial background motion. Perception, 30, 935-944. doi:10.1068/p3132

Ilg, U. J. (1997). Slow eye movements. Progress in Neurobiology, 53, 293-329. doi:10.1016/S03010082(97)00039-7

Itti, L., \& Koch, C. (2000). A saliency-based search mechanism for overt and covert shifts of visual attention. Vision Research, 40, 1489-1506.

Jie, L., \& Clark, J. J. (2008). Video game design using an eye-movement-dependent model of visual attention. ACM Transactions on Multimedia Computing Communications and Applications, 
4(3), article 22, 1-16. doi:10.1145/1386109.1386115

Kaminiarz, A., Krekelberg, B., \& Bremmer, F. (2007). Localization of visual targets during optokinetic eye movements. Vision Research, 47, 869-878. doi:10.1016/j.visres.2006.10.015

Kim, J., \& Palmisano, S. (2010). Eccentric gaze dynamics enhance vection in depth. Journal of Vision, 10(12), article 7, 1-11. doi:10.1167/10.12.7

Neider, M. B., \& Zelinsky, G. J. (2011). Cutting through the clutter: Searching for targets in evolving complex scenes. Journal of Vision, 11(14), article 7, 1-16. doi:10.1167/11.14.7

Rayner, K. (2009). Eye movements and attention in reading, scene perception, and visual search. Quarterly Journal of Experimental Psychology, 62, 1457-1506. doi:10.1080/17470210902816461

Riecke, B. E., Schulte-Pelkum, J., Avraamides, M. N., Von Der Heyde, M., \& Bülthoff, H. H. (2006). Cognitive factors can influence self-motion perception (vection) in virtual reality. ACM Transactions on Applied Perception, 3, 194-216. doi:10.1145/1166087.1166091

Tozzi, A., Morrone, M. C., \& Burr, D. C. (2007). The effect of optokinetic nystagmus on the perceived position of briefly flashed targets. Vision Research, 47, 861-868. doi:10.1016/j.visres.2006.10.022

Trutoiu, L. C., Mohler, B. J., Schulte-Pelkum, J., \& Bülthoff, H. H. (2009). Circular, linear, and curvilinear vection in a large-screen virtual environment with floor projection. Computers \& Graphics, 33, 47-58. doi:10.1016/j.cag.2008.11.008

Waespe, W., \& Schwarz, U. (1987). Slow eye movements induced by apparent target motion in monkey. Experimental Brain Research, 67, 433-435. doi:10.1007/bf00248564

Walther, D., \& Koch, C. (2006). Modeling attention to salient proto-objects. Neural Networks, 19, 1395-1407. doi:10.1016/j.neunet.2006.10.001 
Wolfe, J. M. (1994). Guided Search 2.0: A revised model of visual search. Psychonomic Bulletin \& Review, 1, 202-238. doi:10.3758/bf03200774

Wolfe, J. M., Oliva, A., Horowitz, T. S., Butcher, S. J., \& Bompas, A. (2002). Segmentation of objects from backgrounds in visual search tasks. Vision Research, 42, 2985-3004. doi:10.1016/S00426989(02)00388-7 
Table 1. Means (M) and standard deviations (SD) of dependent measures according to background complexity and motion in Experiment 1.

\begin{tabular}{|c|c|c|c|c|c|c|}
\hline & \multicolumn{2}{|c|}{ No clutter } & \multicolumn{2}{|c|}{ Low clutter } & \multicolumn{2}{|c|}{ High clutter } \\
\hline & $\mathrm{M}$ & SD & $\mathrm{M}$ & SD & $\mathrm{M}$ & SD \\
\hline \multicolumn{7}{|c|}{ Mean response time (in $\mathrm{ms}$ ) } \\
\hline Static background & 1298 & 262 & 1375 & 292 & 1299 & 257 \\
\hline Moving background & 1384 & 237 & 1409 & 234 & 1419 & 262 \\
\hline \multicolumn{7}{|c|}{ Mean number of fixations } \\
\hline Static background & 1.61 & 0.63 & 1.96 & 1.09 & 1.64 & 0.71 \\
\hline Moving background & 1.98 & 0.86 & 2.11 & 0.91 & 2.06 & 0.98 \\
\hline \multicolumn{7}{|c|}{ Mean duration of fixations (in ms) } \\
\hline Static background & 258 & 114 & 235 & 84 & 274 & 103 \\
\hline Moving background & 253 & 97 & 234 & 79 & 250 & 77 \\
\hline \multicolumn{7}{|c|}{ Average position of fixations (in pixels) } \\
\hline \multicolumn{7}{|c|}{ Horizontal coordinate $(X)$} \\
\hline Static background & 632 & 49 & 631 & 53 & 626 & 46 \\
\hline Moving background & 642 & 41 & 658 & 43 & 674 & 57 \\
\hline \multicolumn{7}{|l|}{ Vertical coordinate $(Y)$} \\
\hline Static background & 494 & 47 & 476 & 51 & 477 & 50 \\
\hline Moving background & 489 & 51 & 490 & 46 & 495 & 37 \\
\hline \multicolumn{7}{|c|}{ Mean number of saccades } \\
\hline Static background & 2.13 & 0.48 & 2.36 & 0.54 & 2.18 & 0.60 \\
\hline Moving background & 2.49 & 0.60 & 2.60 & 0.62 & 2.55 & 0.83 \\
\hline \multicolumn{7}{|c|}{ Average amplitude of saccades (in degrees) } \\
\hline Static background & 5.35 & 0.83 & 5.02 & 0.77 & 5.06 & 0.56 \\
\hline Moving background & 4.84 & 0.68 & 4.88 & 0.63 & 4.81 & 0.56 \\
\hline
\end{tabular}


Table 2. Results of ANOVAs and planned comparisons performed in Experiment 1.

\begin{tabular}{|c|c|c|c|c|c|}
\hline ANOVA & Planned comparison & $d f$ & F-ratio & $p$-value & $\eta_{p}^{2}$ \\
\hline \multicolumn{6}{|l|}{ Mean response time } \\
\hline \multirow[t]{4}{*}{ Background Complexity } & & 2,42 & 4.87 & $<.05$ & .19 \\
\hline & No vs. Low & 1,21 & 7.66 & $<.05$ & - \\
\hline & No vs. High & 1,21 & 1.02 & .32 & - \\
\hline & Low vs. High & 1,21 & 4.90 & $<.05$ & - \\
\hline Background Motion & & 1,21 & 32.08 & $<.001$ & .60 \\
\hline Background Complexity x Motion & & 2,42 & 2.08 & .14 & - \\
\hline \multicolumn{6}{|l|}{ Mean number of fixations } \\
\hline \multirow[t]{4}{*}{ Background Complexity } & & 2,42 & 3.89 & $<.05$ & .16 \\
\hline & No vs. Low & 1,21 & 7.24 & $<.05$ & - \\
\hline & No vs. High & 1,21 & 0.57 & .46 & - \\
\hline & Low vs. High & 1,21 & 3.02 & .10 & - \\
\hline Background Motion & & 1,21 & 7.57 & $<.05$ & .27 \\
\hline Background Complexity x Motion & & 2,42 & 1.41 & .26 & - \\
\hline \multicolumn{6}{|l|}{ Mean duration of fixations } \\
\hline Background Complexity & & 2,42 & 2.22 & .12 & - \\
\hline Background Motion & & 1,21 & 0.71 & .41 & - \\
\hline Background Complexity x Motion & & 2,42 & 0.68 & .51 & - \\
\hline \multicolumn{6}{|l|}{ Average position of fixations } \\
\hline \multicolumn{6}{|l|}{ Horizontal coordinate $(X)$} \\
\hline Background Complexity & & 2,42 & 3.38 & .06 & - \\
\hline Background Motion & & 1,21 & 48.34 & $<.001$ & .70 \\
\hline \multirow[t]{7}{*}{ Background Complexity x Motion } & & 2,42 & 6.37 & $<.01$ & .23 \\
\hline & Static, No vs. Low & 1,21 & 0.04 & .84 & - \\
\hline & Static, No vs. High & 1,21 & 0.50 & .49 & - \\
\hline & Static, Low vs. High & 1,21 & 0.74 & .40 & - \\
\hline & Moving, No vs. Low & 1,21 & 6.69 & $<.05$ & - \\
\hline & Moving, No vs. High & 1,21 & 26.27 & $<.001$ & - \\
\hline & Moving, Low vs. High & 1,21 & 5.07 & $<.05$ & - \\
\hline \multicolumn{6}{|l|}{ Vertical coordinate $(Y)$} \\
\hline Background Complexity & & 2,42 & 0.57 & .57 & - \\
\hline Background Motion & & 1,21 & 2.33 & .14 & - \\
\hline Background Complexity x Motion & & 2,42 & 2.38 & .11 & - \\
\hline \multicolumn{6}{|l|}{ Mean number of saccades } \\
\hline Background Complexity & & 2,42 & 2.76 & .08 & - \\
\hline Background Motion & & 1,21 & 18.24 & $<.001$ & .47 \\
\hline Background Complexity x Motion & & 2,42 & 0.47 & .63 & - \\
\hline \multicolumn{6}{|l|}{ Average amplitude of saccades } \\
\hline Background Complexity & & 2,42 & 1.68 & .20 & - \\
\hline Background Motion & & 1,21 & 10.93 & $<.01$ & .34 \\
\hline Background Complexity x Motion & & 2,42 & 2.32 & .11 & - \\
\hline
\end{tabular}

Note. No = No Clutter; Low = Low Clutter; High = High Clutter; Static = Static Background; Moving = Moving Background. Planned comparisons are displayed only when the corresponding main effect or interaction was significant. Partial $\eta^{2}$ are displayed only when the main effects or interactions were significant. 
Table 3. Means (M) and standard deviations (SD) of dependent measures according to background complexity and motion in Experiment 2.

\begin{tabular}{|c|c|c|c|c|c|c|}
\hline & \multicolumn{2}{|c|}{ No clutter } & \multicolumn{2}{|c|}{ Low clutter } & \multicolumn{2}{|c|}{ High clutter } \\
\hline & $\mathrm{M}$ & SD & $M$ & SD & $M$ & SD \\
\hline \multicolumn{7}{|c|}{ Mean response time (in $\mathrm{ms}$ ) } \\
\hline Static background & 1277 & 248 & 1276 & 258 & 1320 & 246 \\
\hline Moving background & 1314 & 229 & 1412 & 297 & 1354 & 250 \\
\hline \multicolumn{7}{|c|}{ Mean number of fixations } \\
\hline Static background & 1.38 & 0.53 & 1.40 & 0.59 & 1.47 & 0.66 \\
\hline Moving background & 1.50 & 0.56 & 1.44 & 0.65 & 1.60 & 0.80 \\
\hline \multicolumn{7}{|c|}{ Mean duration of fixations (in ms) } \\
\hline Static background & 236 & 113 & 247 & 125 & 211 & 86 \\
\hline Moving background & 231 & 69 & 204 & 48 & 204 & 73 \\
\hline \multicolumn{7}{|c|}{ Average position of fixations (in pixels) } \\
\hline \multicolumn{7}{|c|}{ Horizontal coordinate $(X)$} \\
\hline Static background & 644 & 55 & 633 & 64 & 628 & 60 \\
\hline Moving background & 652 & 56 & 663 & 54 & 648 & 58 \\
\hline \multicolumn{7}{|c|}{ Vertical coordinate $(Y)$} \\
\hline Static background & 470 & 77 & 498 & 53 & 479 & 59 \\
\hline Moving background & 480 & 52 & 480 & 63 & 491 & 51 \\
\hline \multicolumn{7}{|c|}{ Mean number of saccades } \\
\hline Static background & 1.87 & 0.63 & 1.81 & 0.76 & 1.95 & 0.82 \\
\hline Moving background & 2.00 & 0.64 & 1.89 & 0.80 & 1.98 & 0.95 \\
\hline \multicolumn{7}{|c|}{ Average amplitude of saccades (in degrees) } \\
\hline Static background & 5.70 & 1.08 & 5.61 & 1.04 & 6.04 & 1.57 \\
\hline Moving background & 5.23 & 0.98 & 5.70 & 1.05 & 5.51 & 1.36 \\
\hline
\end{tabular}


Table 4. Results of ANOVAs and planned comparisons performed in Experiment 2.

\begin{tabular}{|c|c|c|c|c|c|}
\hline ANOVA & Planned comparison & $d f$ & F-ratio & $p$-value & $\eta_{p}^{2}$ \\
\hline \multicolumn{6}{|l|}{ Mean response time } \\
\hline Background Complexity & & 2,42 & 2.65 & .08 & - \\
\hline Background Motion & & 1,21 & 6.71 & $<.05$ & .70 \\
\hline \multirow[t]{7}{*}{ Background Complexity x Motion } & & 2,42 & 3.81 & $<.05$ & .66 \\
\hline & Static, No vs. Low & 1,21 & 0.01 & .97 & - \\
\hline & Static, No vs. High & 1,21 & 3.56 & .07 & - \\
\hline & Static, Low vs. High & 1,21 & 2.87 & .10 & - \\
\hline & Moving, No vs. Low & 1,21 & 6.97 & $<.05$ & - \\
\hline & Moving, No vs. High & 1,21 & 1.88 & .18 & - \\
\hline & Moving, Low vs. High & 1,21 & 3.07 & .09 & - \\
\hline \multicolumn{6}{|l|}{ Mean number of fixations } \\
\hline Background Complexity & & 2,42 & 1.01 & .37 & - \\
\hline Background Motion & & 1,21 & 1.92 & .18 & - \\
\hline Background Complexity x Motion & & 2,42 & 0.32 & .73 & - \\
\hline \multicolumn{6}{|l|}{ Mean duration of fixations } \\
\hline Background Complexity & & 2,42 & 1.40 & .26 & - \\
\hline Background Motion & & 1,21 & 1.76 & .20 & - \\
\hline Background Complexity x Motion & & 2,42 & 1.14 & .33 & - \\
\hline \multicolumn{6}{|l|}{ Average position of fixations } \\
\hline \multicolumn{6}{|l|}{ Horizontal coordinate $(X)$} \\
\hline Background Complexity & & 2,42 & 0.86 & .43 & - \\
\hline Background Motion & & 1,21 & 20.36 & $<.001$ & .49 \\
\hline Background Complexity x Motion & & 2,42 & 1.55 & .22 & - \\
\hline \multicolumn{6}{|l|}{ Vertical coordinate $(Y)$} \\
\hline Background Complexity & & 2,42 & 2.00 & .15 & - \\
\hline Background Motion & & 1,21 & 0.03 & .87 & - \\
\hline Background Complexity x Motion & & 2,42 & 1.74 & .19 & - \\
\hline \multicolumn{6}{|l|}{ Mean number of saccades } \\
\hline Background Complexity & & 2,42 & 0.90 & .41 & - \\
\hline Background Motion & & 1,21 & 0.96 & .34 & - \\
\hline Background Complexity x Motion & & 2,42 & 0.30 & .74 & - \\
\hline \multicolumn{6}{|l|}{ Average amplitude of saccades } \\
\hline Background Complexity & & 2,42 & 2.20 & .12 & - \\
\hline Background Motion & & 1,21 & 6.05 & $<.05$ & .22 \\
\hline Background Complexity x Motion & & 2,42 & 2.29 & .11 & - \\
\hline
\end{tabular}

Note. No = No Clutter; Low = Low Clutter; High = High Clutter; Static = Static Background; Moving = Moving Background. Planned comparisons are displayed only when the corresponding main effect or interaction was significant. Partial $\eta^{2}$ are displayed only when the main effects or interactions were significant. 
Experiment 1

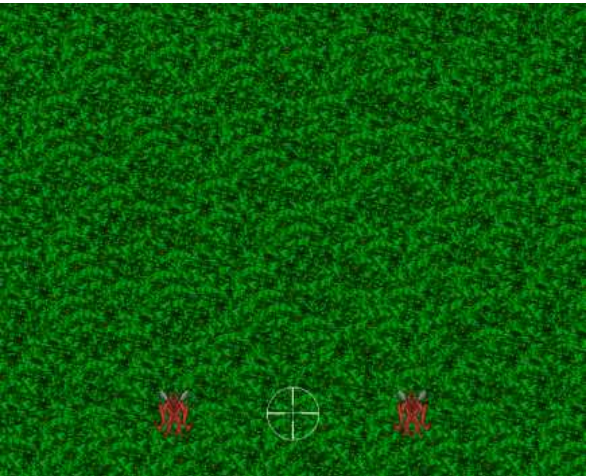

No

clutter

$\frac{7}{x}$
$\frac{0}{0}$
$\frac{0}{8}$
$\frac{0}{0}$
$\frac{0}{3}$
$\frac{0}{00}$
$\frac{0}{0}$
0

clutter
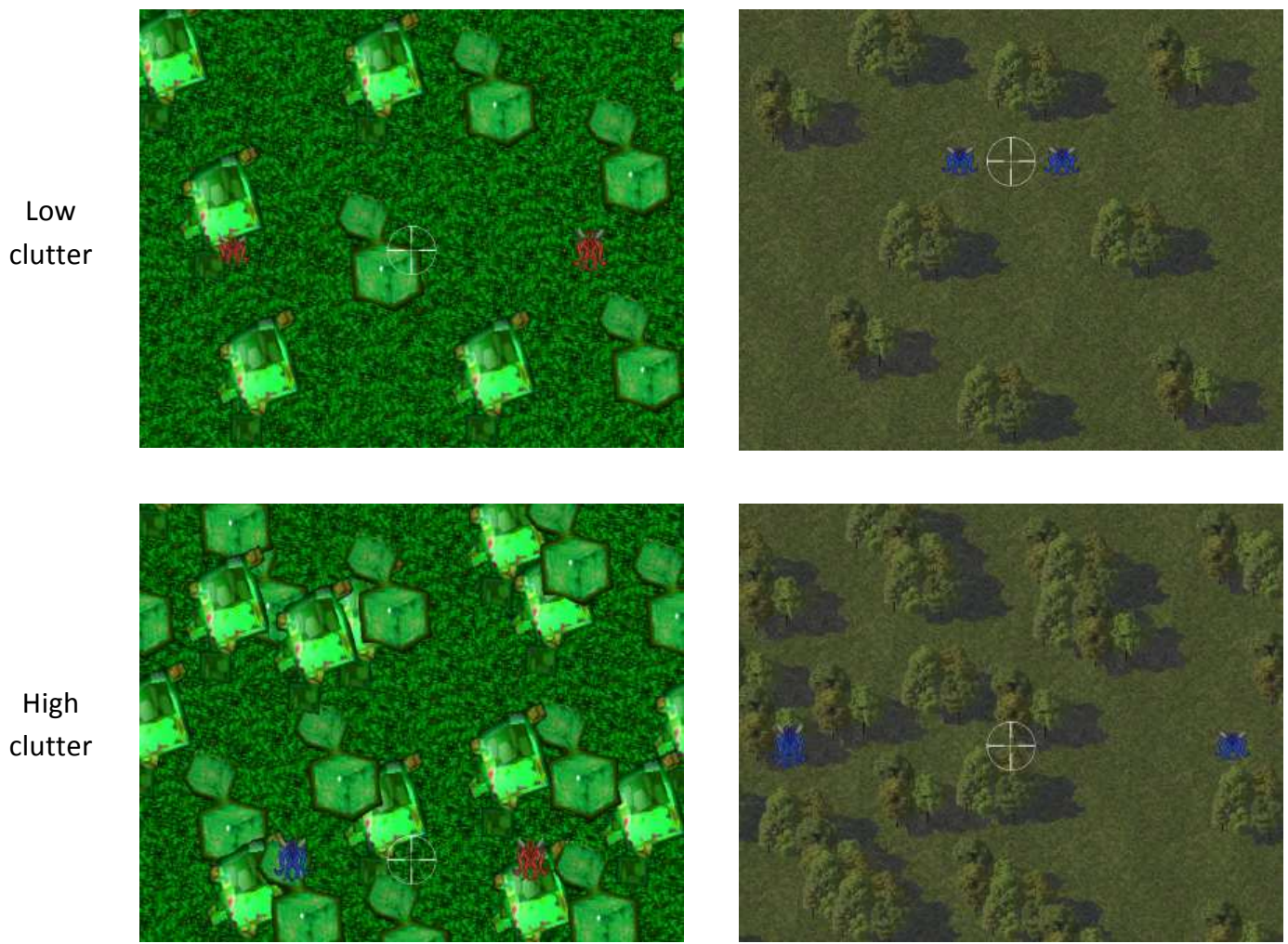

Experiment 2
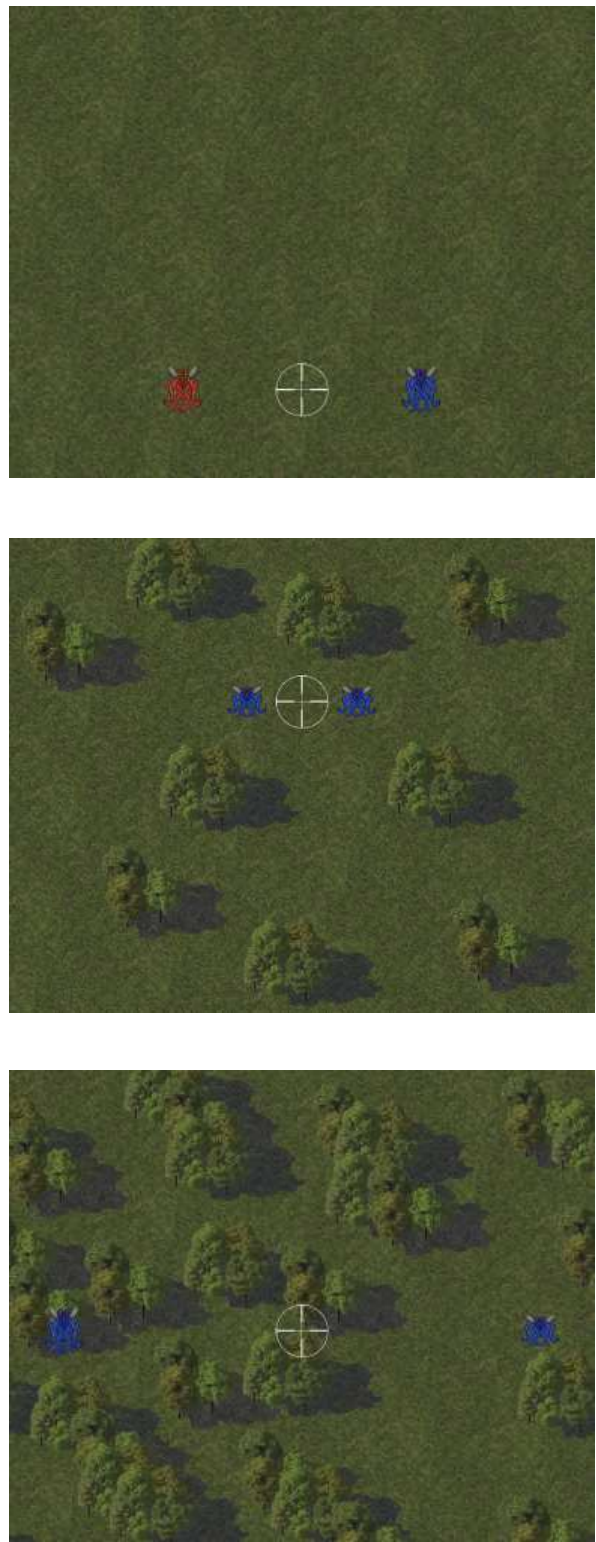

Figure 1. Backgrounds used in Experiment 1 and Experiment 2, with different examples of locations of a target, a distractor and the cursor. For each experiment, three backgrounds of various complexities were designed. The complexity was determined by the clutter, i.e. the proportion of the initial, neutral pattern covered by the superimposed objects. 


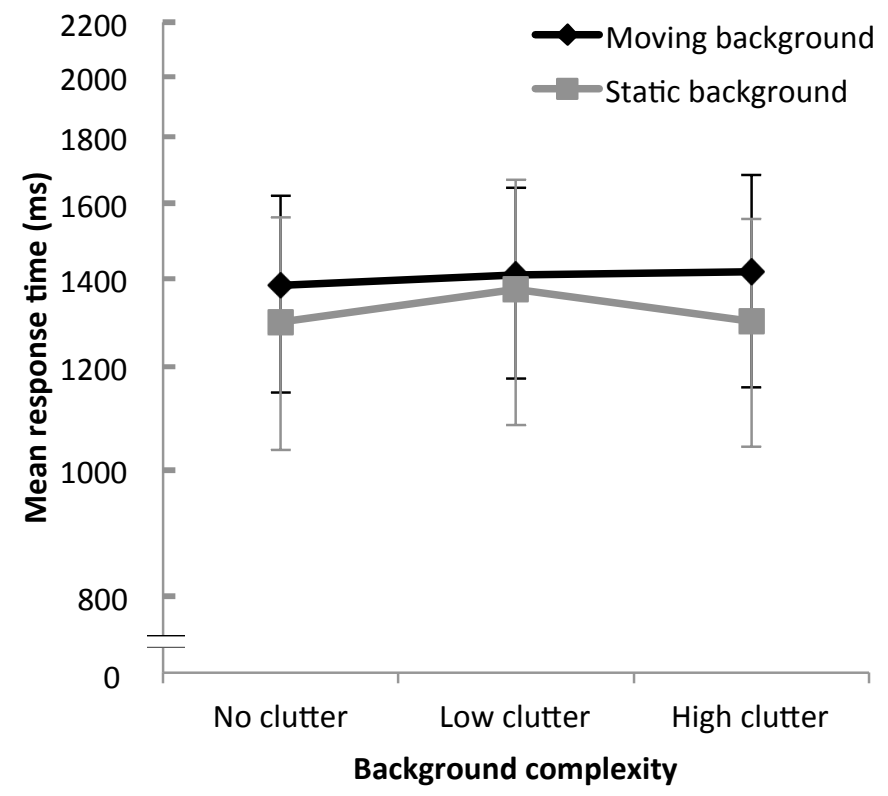

Figure 2. Mean response time (in $\mathrm{ms}$ ) to hit each target in each condition of background complexity and motion in Experiment 1, represented on a logarithmically spaced axis. The error bars represent \pm 1 standard error of the mean. 


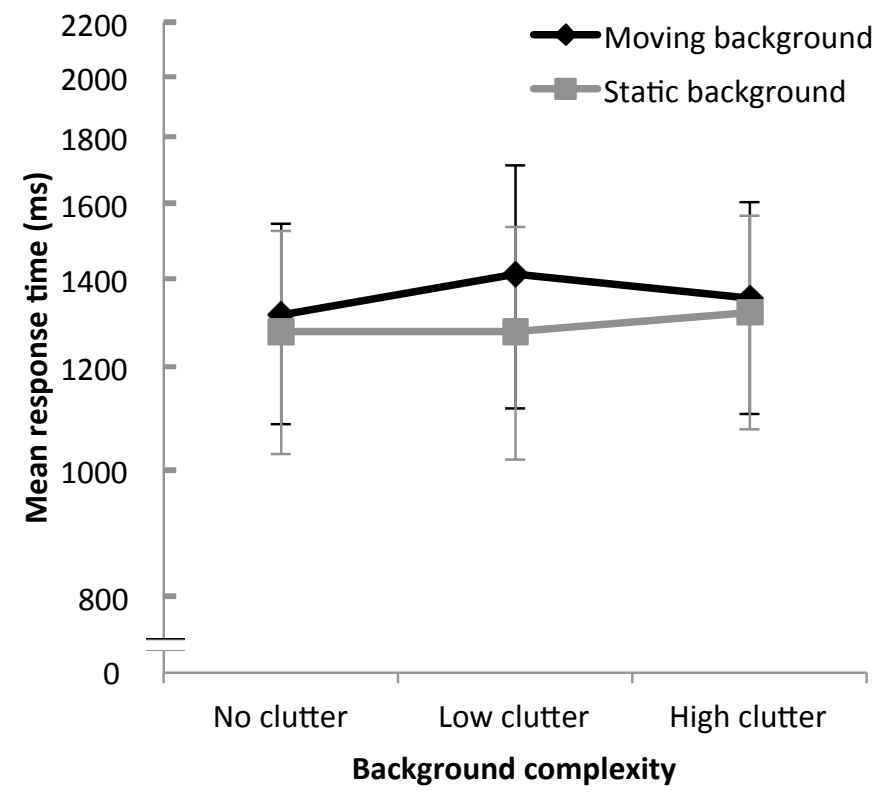

Figure 3. Mean response time (in $\mathrm{ms}$ ) to hit each target within each condition of background complexity and motion in Experiment 2, represented on a logarithmically spaced axis. The error bars represent \pm 1 standard error of the mean. 


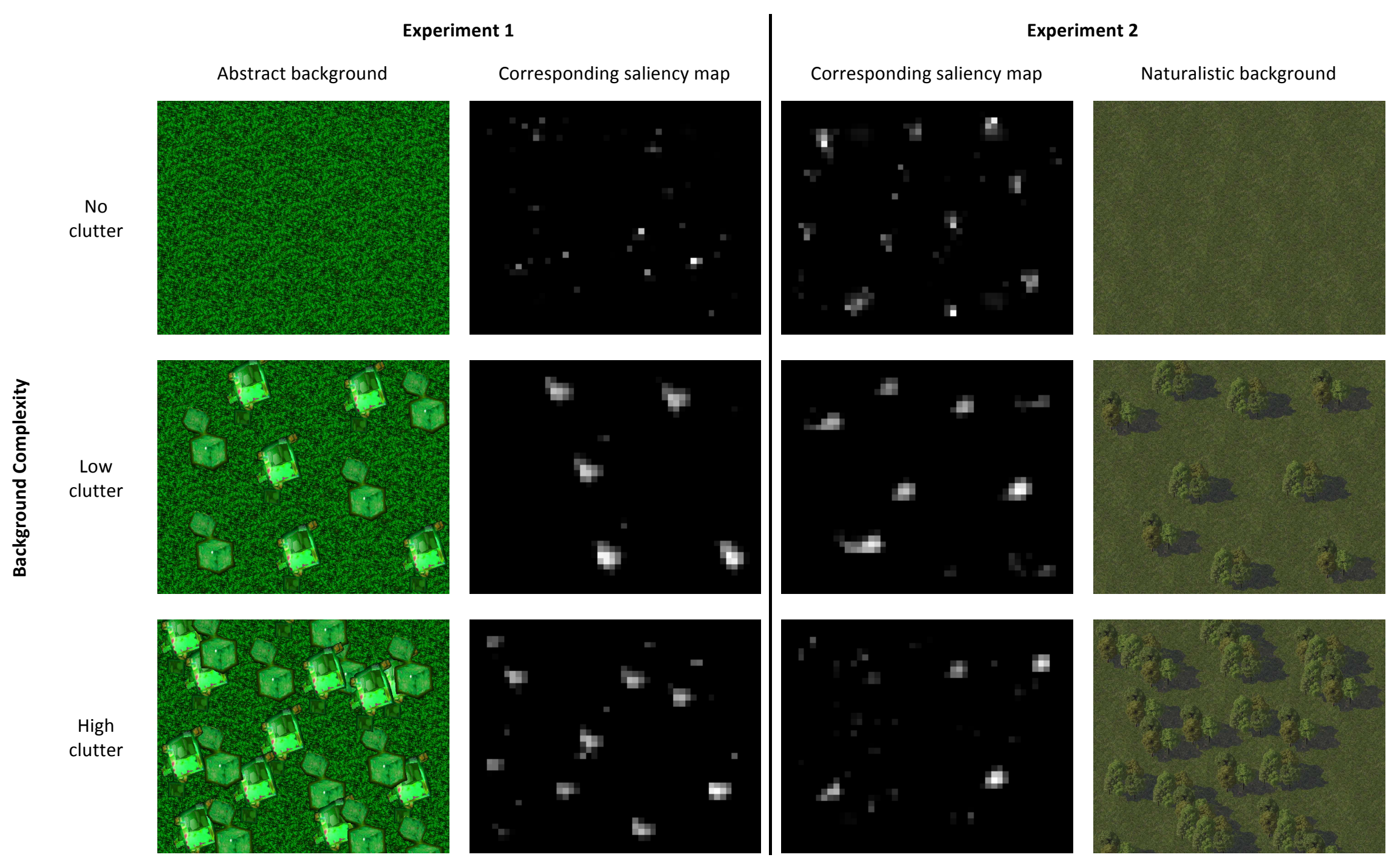

Figure 4. Backgrounds used in Experiments 1 and 2 with the corresponding saliency maps. The brighter regions within the saliency maps indicate higher values of saliency. 